\title{
AN ASYMPTOTIC FORMULA FOR AN INTEGRAL IN STARLIKE FUNCTION THEORY
}

BY

\author{
R. R. LONDON AND D. K. THOMAS
}

ABSTRACT. The paper is concerned with the integral

$$
H=\int_{0}^{2 \pi}|f|^{\sigma}|F|^{\tau}(\operatorname{Re} F)^{\kappa} d \theta
$$

in which $f$ is a function regular and starlike in the unit disc, $F=z f^{\prime} / f$, and the parameters $\sigma, \tau, K$ are real. A study of $H$ is of interest since various wellknown integrals in the theory, such as the length of $f(|z|=r)$, the area of $f(|z|<r)$, and the integral means of $f$, are essentially obtained from it by suitably choosing the parameters. An asymptotic formula, valid as $r \rightarrow 1$, is obtained for $H$ when $f$ is a starlike function of positive order $\alpha$, and the parameters satisfy $\alpha \sigma+\tau+\kappa>1, \tau+\kappa>0, k>0, \sigma>0$. Several easy applications of this result are made; some to obtaining old results, two others in proving conjectures of Holland and Thomas.

1. Introduction. Let a function $f$ be regular in the open unit disc $D$, and such that $f(0)=0, f^{\prime}(0)=1$. Suppose a function $F$ exists, regular in $D$, and of positive real part, for which

$$
F(z)=z f^{\prime}(z) / f(z) \quad(0<|z|<1), \quad F(0)=1 .
$$

Then $f$ is called starlike. It is well known that a starlike function is univalent, and maps $D$ onto a set starshaped with respect to the origin.

Suppose now that $f$ is starlike, and let (1.1) define $F$. We consider for $0<$ $r<1$ and real $\sigma, \tau$, and $\kappa$, the integral

$$
H(r, \sigma, \tau, \kappa)=\int_{0}^{2 \pi}\left|f\left(r e^{i \theta}\right)\right|^{\sigma}\left|F\left(r e^{i \theta}\right)\right|^{r}\left(\operatorname{Re} F\left(r e^{i \theta}\right)\right)^{\kappa} d \theta .
$$

With various choices of $\sigma, \tau$, and $\kappa$, this integral is well known in starlike (and univalent) function theory. For example,

$$
H(r, 1,1,0)=\int_{0}^{2 \pi}\left|f\left(r e^{i \theta}\right) F\left(r e^{i \theta}\right)\right| d \theta=\int_{0}^{2 \pi} r\left|f^{\prime}\left(r e^{i \theta}\right)\right| d \theta
$$

is the length of $f(\{z:|z|=r\})$, and

$$
\begin{aligned}
H(r, 2,0,1) & =\int_{0}^{2 \pi}\left|f\left(r e^{i \theta}\right)\right|^{2} \operatorname{Re} F\left(r e^{i \theta}\right) d \theta \\
& =2 \int_{0}^{r} \int_{0}^{2 \pi} \rho\left|f^{\prime}\left(\rho e^{i \theta}\right)\right|^{2} d \theta d \rho=2 A(r, f),
\end{aligned}
$$

Received by the editors November 22, 1974.

AMS (MOS) subject classifications (1970). Primary 30A32; Secondary 30A32.

Key words and phrases. Starlike function, order, length, area, integral means. 
where $A(r, f)$ is the area of $f(\{z:|z| \leqslant r\})$. Also, for $\lambda>0$,

$$
H(r, \lambda, 0,0)=\int_{0}^{2 \pi}\left|f\left(r e^{i \theta}\right)\right|^{\lambda} d \theta=2 \pi I(r, \lambda, f),
$$

and

$$
\begin{aligned}
H(r, \lambda, \lambda, 0) & =\int_{0}^{2 \pi}\left|f\left(r e^{i \theta}\right) F\left(r e^{i \theta}\right)\right|^{\lambda} d \theta \\
& =\int_{0}^{2 \pi} r^{\lambda}\left|f^{\prime}\left(r e^{i \theta}\right)\right|^{\lambda} d \theta=2 \pi r^{\lambda} J(r, \lambda, f),
\end{aligned}
$$

where $(I(r, \lambda, f))^{1 / \lambda}$ and $(J(r, \lambda, f))^{1 / \lambda}$ are integral means of $f$ and $f^{\prime}$ respectively. In the present paper we suppose $f$ to have a certain minimal growth, and find, for suitable $\sigma, \tau$, and $\kappa$, as $r$ tends to one, an asymptotic formula for $H(r, \sigma, \tau, \kappa)$.

A few remarks and definitions precede the statement of this result. From a classical theorem on regular functions of positive real part, and the relationship (1.1), we find for a starlike function $f$ the well-known representation

$$
f(z)=z \exp \left(-\int_{0}^{2 \pi} \log \left(1-z e^{-i t}\right) d \mu(t)\right) \quad(z \in D),
$$

where $\mu$ is nondecreasing on $[0,2 \pi]$ and $\int_{0}^{2 \pi} d \mu(t)=2$. Any such function $\mu$ satisfying (1.5) is continuous apart from jumps of height at most $\int_{0}^{2 \pi} d \mu(t)$. Pommerenke has shown (implicitly) in [4] that

$$
\Delta(\varphi, f)=\lim _{r \rightarrow 1} \frac{\log \left|f\left(r e^{i \theta}\right)\right|}{-\log (1-r)}
$$

is the jump of $\mu$ at $\varphi$ if $0<\varphi<2 \pi$, and the sum of the jumps at 0 and $2 \pi$ when $\varphi=0$ or $2 \pi$. For $\alpha(f)=\sup _{\varphi} \Delta(\varphi, f)$ and $M(r)=\max _{|z|=r}|f(z)|(0<r<1)$ he has shown

$$
\alpha(f)=\lim _{r \rightarrow 1} \frac{\log M(r, f)}{-\log (1-r)} .
$$

We call $\alpha(f)$ the order of $f$, and $\Delta(\varphi, f)$ the radial order of $f$ on $\left\{r e^{i \varphi}\right\}$.

Starlike functions of positive order are the main concern of the present paper. We shall, in the following, make implicit use of the fact that for such a function $f,\{\varphi: \Delta(\varphi, f)>0\}$ is countable; and for $0<c \leqslant \alpha,\{\varphi: \Delta(\varphi, f) \geqslant c\}$ is finite nonempty.

Our result is as follows:

THEOREM 1. Let $f$ be a starlike function of positive order $\alpha$, and denote by $\varphi_{1}, \ldots, \varphi_{N}$ the values of $\varphi$ in $[0,2 \pi)$ for which $\alpha$ is the radial order of $f$ on $\left\{r e^{i \varphi}\right\}$. Then if $\sigma>0, \kappa \geqslant 0, \tau+\kappa \geqslant 0$, and $\alpha \sigma+\tau+\kappa>1$,

$$
H(r, \sigma, \tau, \kappa) \sim \alpha^{\tau+\kappa} C(\alpha \sigma+\tau+2 \kappa)(1-r)^{1-\tau-\kappa} \sum_{\nu=1}^{N}\left|f\left(r e^{i \varphi_{\nu}}\right)\right|^{\sigma},
$$


as $r \rightarrow 1$, where, for $x>1, C(x)=\int_{-\infty}^{\infty} d t /\left(1+t^{2}\right)^{1 / 2 x}=\Gamma(1 / 2 x-1 / 2) \Gamma(1 / 2) / \Gamma(1 / 2 x)$.

Theorem 1 has some interesting applications. Let $f$ be starlike of order $\alpha$. Recalling (1.2) to (1.4), and using, with Theorem 1, the relationship

$$
M(r, f) \sim \max \left(\left|f\left(r e^{i \varphi_{1}}\right)\right|, \ldots,\left|f\left(r e^{i \varphi_{N}}\right)\right|\right) \quad(\alpha>0)
$$

(proved at the end of $\S 2$ ) we obtain for $\alpha>0$ :

$$
\liminf _{r \rightarrow 1} \frac{A(r, f)}{M^{2}(r, f)} \geqslant \frac{1}{2} \alpha C(2 \alpha+2), \quad \limsup _{r \rightarrow 1} \frac{A(r, f)}{M^{2}(r, f)} \leqslant \frac{1}{2} N \alpha C(2 \alpha+2),
$$

for $\alpha>0, \alpha \lambda>1$ :

$\liminf _{r \rightarrow 1} \frac{I(r, \lambda, f)}{(1-r) M^{\lambda}(r, f)} \geqslant C(\alpha \lambda), \quad \limsup _{r \rightarrow 1} \frac{I(r, \lambda, f)}{(1-r) M^{\lambda}(r, f)} \leqslant N C(\alpha \lambda)$,

and for $\alpha>0,(1+\alpha) \lambda>1$ :

$$
\begin{aligned}
& \liminf _{r \rightarrow 1} \frac{J(r, \lambda, f)}{(1-r)^{1-\lambda} M^{\lambda}(r, f)} \geqslant \alpha^{\lambda} C(\alpha \lambda+\lambda), \\
& \limsup _{r \rightarrow 1} \frac{J(r, \lambda, f)}{(1-r)^{1-\lambda} M^{\lambda}(r, f)} \leqslant N \alpha^{\lambda} C(\alpha \lambda+\lambda) .
\end{aligned}
$$

These are all results of Sheil-Small [5].

From (1.2), $A(r, f)=1 / 2 H(r, 2,0,1)$ and $A^{\prime}(r, f)=H(r, 2,2,0) / r$; so for $\alpha>0$ Theorem 1 also yields

$$
\lim _{r \rightarrow 1} \frac{(1-r) A^{\prime}(r, f)}{A(r, f)}=2 \alpha \text {. }
$$

A proof of this result, and a simple proof of (1.8) in the case $\alpha=0$, are to be found in [3]. We are also able to prove, using Theorem 1, that, for $\alpha \lambda>1$,

$$
\lim _{r \rightarrow 1} \frac{(1-r) I^{\prime}(r, \lambda, f)}{I(r, \lambda, f)}=\alpha \lambda-1,
$$

a result conjectured in [2]. In fact, once we have noticed, from (1.3), that, for any $\lambda>0, I(r, \lambda, f)=H(r, \lambda, 0,0) / 2 \pi$, and

$$
I^{\prime}(r, \lambda, f)=\frac{\lambda}{2 \pi r} \int_{0}^{2 \pi}\left|f\left(r e^{i \theta}\right)\right|^{\lambda} \operatorname{Re} F\left(r e^{i \theta}\right) d \theta=\frac{\lambda}{2 \pi r} H(r, \lambda, 0,1),
$$

the proof via Theorem 1 follows simply on noting

$$
\alpha \lambda C(\alpha \lambda+2)=(\alpha \lambda-1) C(\alpha \lambda), \text { for } \alpha \lambda>1 .
$$

Another conjecture in [2] is that, for $(1+\alpha) \lambda>1$,

$$
\lim _{r \rightarrow 1} \frac{(1-r) J^{\prime}(r, \lambda, f)}{J(r, \lambda, f)}=(1+\alpha) \lambda-1 \text {. }
$$

A corollary of Theorem 1 yields a proof of $(1.10)$ when $(1+\alpha) \lambda>1, \alpha>0$, as we shall see in $\$ 4$.

Our proof of Theorem 1 begins in $\S 2$, where some preliminary results are 
obtained, and is completed in $\S 3$. In all that follows we assume that $0<r<1$, and that $\theta$ is a real number. Also that the 0,0 , and $\sim$ notations refer to behaviour as $r$ tends to one. The term $r$ near one means all values of $r$ in $(\eta, 1)$, for some $\eta$ in $(0,1)$.

2. Preliminaries. In this section we prove a number of results on what are essentially powers of starlike functions. A function $g$ will be called star-powered whenever

$$
g(z)=z \exp \left(-\int_{0}^{2 \pi} \log \left(1-e^{-i t} z\right) d \nu(t)\right) \quad(z \in D),
$$

for some nondecreasing function $\nu$ on $[0,2 \pi]$. For such a $g$, we define as for a starlike function $g$ the terms order of $g$ and radial order of $g$ on $\left\{r e^{i \varphi}\right\}$.

The results we now prove are directed towards finding for a star-powered function $g$, and the function

$$
G: G(z)=z g^{\prime}(z) / g(z) \quad(z \in D \backslash\{0\}), \quad G(0)=1
$$

information about behaviour on various subsets of $D$.

LEMMA 2.1. Let $g$ be a star-powered function of positive order $\beta$, and in the above notation, put $K=\int_{0}^{2 \pi} d \nu(t)$. Then

(i) for $\epsilon>0$, and $r$ near one, $M(r, g)<(1-r)^{-\beta-\epsilon}$;

(ii) with $G$ defined by $(2.2), M(r, G) \leqslant 1+K r(1-r)^{-1}$.

This is a well-known result when $g$ is starlike [4], [3] and the extension to star-powered functions is simple enough to omit.

LEMMA 2.2. Let $g$ be a star-powered function of positive order $\beta$, and denote by $\psi_{1}, \ldots, \psi_{p}$ the values of $\psi$ in $[0,2 \pi)$ for which $\beta$ is the radial order of $f$ on $\left\{r e^{i \varphi}\right\}$. Put

$$
T(r)=[0,2 \pi] \backslash \bigcup_{k=1}^{p}\left\{\theta \bmod 2 \pi:\left|\theta-\psi_{k}\right|<l(r)\right\},
$$

where $l(r)=(-\log 1-r)^{-1}$, and let $\gamma$ be the largest radial order of $g$ less than $\beta$. Then for any positive $\epsilon$, and $r$ near one,

$$
\sup _{\theta \in T(r)}\left|g\left(r e^{i \theta}\right)\right|=O(1)(1-r)^{-\gamma-\epsilon} .
$$

Proof. The function $h$,

$$
h(z)=g(z) \prod_{k=1}^{p}\left(1-z e^{-i \psi k}\right)^{\beta},
$$

is star-powered and has order $\gamma$, so by Lemma 2.1, for $\epsilon>0$, and $r$ near one, $M(r, h)<(1-r)^{-\gamma-1 / 2 \epsilon}$. Also, for $r$ near one, we have uniformly when $\theta \in T(r)$

$$
\left.\prod_{k=1}^{p} \mid 1-r e^{i(\theta-\psi k}\right)^{-\beta} \leqslant \mid 1-r e^{i l(r) \mid-\beta p} \leqslant\left(1 / 2 l^{2}(r)\right)^{-1 / 2 \beta p}
$$


Hence

$$
\sup _{\theta \in T(r)}\left|g\left(r e^{i \theta}\right)\right|=O(1)(1-r)^{-\gamma-1 / 2 \epsilon}(-\log (1-r))^{\beta p},
$$

from which the stated result follows.

LEMMA 2.3. Let $g$ be a star-powered function of positive radial order $\beta$ on $\left\{r e^{i \varphi}\right\} \quad(0 \leqslant \varphi<2 \pi)$. Suppose that $\lambda$ is a positive number, and $\delta$ a positive function on $(0,1)$ for which $\lambda(1-r)<\delta(r)=o(1)$. Then

$$
\left|g\left(r e^{i \theta}\right)\right|<\left(1+\lambda^{2}+o(1)\right)^{-1 / 2 \beta}\left|g\left(r e^{i \varphi}\right)\right|
$$

uniformly when $\lambda(1-r)<|\theta-\varphi|<\delta(r)$.

Proof. Let

$$
g(z)=z h(z)\left(1-z e^{-i \varphi}\right)^{-\beta} \quad(z \in D)
$$

then, by an elementary argument,

$$
\left|g\left(r e^{i \theta}\right)\right|<(1+o(1)) r\left|h\left(r e^{i \theta}\right)\right|(1-r)^{-\beta}\left(1+\left(\frac{\theta-\varphi}{1-r}\right)^{2}\right)^{-1 / 2 \beta}
$$

uniformly when $|\theta-\varphi|<\delta(r)$. For $h$ we shall prove

$$
\left|h\left(r e^{i \theta}\right)\right|<\left|h\left(r e^{i \varphi}\right)\right| \exp \left(o(1) \int_{0}^{|\theta-\varphi| / 2(1-r)}\left(1+t^{2}\right)^{-1 / 2} d t\right)
$$

uniformly when $\lambda(1-r)<|\theta-\varphi|<\delta(r)$. The lemma then follows by combining (2.3) and (2.4) to form

$$
\begin{aligned}
\left|g\left(r e^{i \theta}\right)\right|< & (1+o(1))\left|g\left(r e^{i \varphi}\right)\right|\left(1+\left(\frac{\theta-\varphi}{1-r}\right)^{2}\right)^{-1 / 2 \beta} \\
& \cdot \exp \left(o(1) \int_{0}^{(\theta-\varphi \mid / 2(1-r)}\left(1+t^{2}\right)^{-1 / 2} d t\right),
\end{aligned}
$$

valid uniformly for $\lambda(1-r)<|\theta-\varphi|<\delta(r)$, and by noting that for any suitably small positive $\epsilon$

$$
y_{\epsilon}: y_{\epsilon}(x)=\left(1+x^{2}\right)^{-1 / 2 \beta} \exp \left(\epsilon \int_{0}^{1 / 2 x}\left(1+t^{2}\right)^{-1 / 2} d t\right) \quad(x>\lambda)
$$

is a decreasing function.

For the proof of $(2.5)$, let $\eta$ be a positive function on $(0,1)$ for which $\eta(r)=o(1)$, yet $\delta(r)=o(1) \mid 1-r e^{i \eta(r) \mid}$, and put

$$
E(r)=\{\theta \bmod 2 \pi:|\theta-\varphi|<\eta(r)\}, \quad E^{\prime}(r)=[0,2 \pi] \backslash E(r) .
$$

Write

$$
g(z)=z \exp \left(-\int_{0}^{2 \pi} \log \left(1-e^{-i t} z\right) d \nu(t)\right) \quad(z \in D),
$$

then from (2.3) we deduce

$$
h(z)=\exp \left(-\int_{0}^{2 \pi} \log \left(1-e^{-i t} z\right) d \tau(t)\right) \quad(z \in D)
$$


where

$$
\tau(t)=v(t)- \begin{cases}\beta, & \varphi<t \leqslant 2 \pi \\ 0, & 0 \leqslant t \leqslant \varphi\end{cases}
$$

We have, for any real $t$,

$$
\begin{aligned}
\log \mid 1-r e^{i(\varphi-t) \mid}- & \log \mid 1-r e^{i(\theta-t) \mid}=\operatorname{Re} \int_{\varphi-t}^{\theta-t} \frac{\partial}{\partial u}\left(\log \frac{1}{1-r e^{i u}}\right) d u \\
& \leqslant \max _{0<t<2 \pi} \int_{t}^{t+|\theta-\varphi|} \frac{d u}{\mid \mathrm{i}-r e^{i u \mid}}=2 \int_{0}^{1 / 2|\theta-\varphi|} \frac{d u}{\mid 1-r e^{i u \mid}} .
\end{aligned}
$$

So with the aid of (2.7) we obtain

$$
\begin{aligned}
& \int_{E(r)}\left(\log \mid 1-r e^{\left.i(\varphi-t)|-\log | 1-r e^{i(\theta-t)} \mid\right) d \tau(t)}\right. \\
& \quad \leqslant 2\left(\int_{E(r)} d \tau(t)\right)\left(\int_{0}^{1 / 2|\theta-\varphi|} \frac{d u}{\mid 1-r e^{i u \mid}}\right) \\
& \quad=o(1) \int_{0}^{1 / 2|\theta-\varphi|} \frac{d u}{\mid 1-r e^{i u \mid}}=o(1) \int_{0}^{\mid \theta-\varphi V 2(1-r)}\left(1+u^{2}\right)^{-1 / 2} d u,
\end{aligned}
$$

uniformly when $|\theta-\varphi|<\delta(r)$. Now observe that, by the choice of $\eta$,

$$
\left|\frac{1-r e^{i(\theta-t)}}{1-r e^{i(\varphi-t)}}-1\right| \leqslant\left|\frac{e^{i(\theta-\varphi)}-1}{1-r e^{i \eta(r)}}\right|=o(1)
$$

uniformly for $t \in E^{\prime}$ and $|\theta-\varphi|<\delta(r)$. Hence

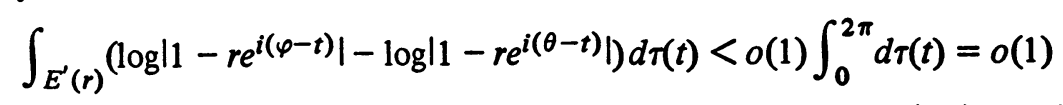

uniformly when $|\theta-\varphi|<\delta(r)$. Combining (2.8) and (2.9), and using (2.6) we obtain (2.5) uniformly for $\lambda(1-r)<|\theta-\varphi|<\delta(r)$.

LEMMA 2.4. Let $g$ be a star-powered function of positive radial order $\beta$ on $\left\{r e^{i \varphi}\right\}(0 \leqslant \varphi<2 \pi)$, and define $G$ by (2.2). Then for $0<c<C$

$$
\left|G\left(r e^{i \theta}\right)\right| \sim \beta(1-r)^{-1}\left(1+\left(\frac{\theta-\varphi}{1-r}\right)^{2}\right)^{-1 / 2},
$$

and

$$
\operatorname{Re} G\left(r e^{i \theta}\right) \sim \beta(1-r)^{-1}\left(1+\left(\frac{\theta-\varphi}{1-r}\right)^{2}\right)^{-1},
$$

uniformly when $|\theta-\varphi|<C(1-r)$.

(ii)

$$
\operatorname{Im} G\left(r e^{i \theta}\right) \sim \beta(\theta-\varphi)(1-r)^{-2}\left(1+\left(\frac{\theta-\varphi}{1-r}\right)^{2}\right)^{-1}
$$


uniformly when $c(1-r)<|\theta-\varphi|<C(1-r)$.

(iii)

$$
\left|g\left(r e^{i \theta}\right)\right| \sim\left|g\left(r e^{i \varphi}\right)\right|\left(1+\left(\frac{\theta-\varphi}{1-r}\right)^{2}\right)^{-1 / 2 \beta}
$$

uniformly when $|\theta-\varphi|<C(1-r)$.

Proof. Parts (i) and (ii) are easily proved if $G(z)=\beta z /(1-z), \varphi=0$, so in deriving (i) and (ii) we shall prove no more than

$$
\left|G\left(r e^{i \theta}\right)-\frac{\beta r e^{i(\theta-\varphi)}}{1-r e^{i(\theta-\varphi)}}\right|=\frac{o(1)}{1-r}
$$

uniformly when $|\theta-\varphi|<\delta(r)$, where $\delta$ is any positive $o(1)$ function on $(0,1)$. We have, from (2.1) and (2.2), for some nondecreasing function $\nu$,

$$
G(z)=1+\int_{0}^{2 \pi} \frac{e^{-i t_{z}}}{1-e^{-i t_{z}}} d \nu(t) \quad(z \in D)
$$

and this we rewrite in terms of

$$
\tau(t)=\nu(t)- \begin{cases}\beta, & \varphi<t \leqslant 2 \pi \\ 0, & 0 \leqslant t \leqslant \varphi\end{cases}
$$

as

$$
G\left(r e^{i \theta}\right)-\frac{\beta r e^{i(\theta-\varphi)}}{1-r e^{i(\theta-\varphi)}}=1+\int_{0}^{2 \pi} \frac{r e^{i(\theta-t)}}{1-r e^{i(\theta-t)}} d \tau(t) .
$$

Let $\eta$ and $\epsilon$ be positive functions on $(0,1)$ for which $\epsilon(r)=\delta(r)+\eta(r)=o(1)$, and $1-r=o(1)\left|1-r e^{i \eta(r)}\right|$. Put

$$
P(r)=\{\theta \bmod 2 \pi:|\theta-\varphi|<\epsilon(r)\}, \quad Q(r)=[0,2 \pi] \backslash P(r),
$$

and consider now only values of $r$ for which $Q(r)$ is nonempty. Then from (2.11) we see that

$$
\left|\int_{P(r)} \frac{r e^{i(\theta-t)}}{1-r e^{i(\theta-t)}} d \tau(t)\right| \leqslant \frac{1}{1-r} \int_{P(r)} d \tau(t)=\frac{o(1)}{1-r}
$$

uniformly for real $\theta$. Moreover, for $|\theta-\varphi|<\delta(r)$ and $t \in Q(r)$, $\eta(r) \leqslant|\theta-t| \leqslant 2 \pi-\eta(r)$, so we also have, uniformly for $|\theta-\varphi|<\delta(r)$,

$$
\left|\int_{Q(r)} \frac{r e^{i(\theta-t)}}{1-r e^{i(\theta-t)}} d \tau(t)\right| \leqslant\left|\frac{1}{1-r e^{i \eta(r)}}\right| \int_{Q(r)} d \tau(t)=\frac{o(1)}{1-r},
$$

by the choice of $\eta$. Now combining (2.12), (2.13) and (2.14), the required estimate (2.10) is easily obtained.

We next derive (iii). Using the identity

$$
\frac{\partial}{\partial \theta} \log \lg \left(r e^{i \theta}\right) \mid=-\operatorname{Im} G\left(r e^{i \theta}\right)
$$


and (ii) of this lemma, we have, uniformly for $c(1-r)<\theta-\varphi<C(1-r)$,

$$
\begin{aligned}
\log \left|\frac{g\left(r e^{i \theta}\right)}{g\left(r e^{i(\varphi+c(1-r))}\right)}\right| & \sim-\beta \int_{\varphi+c(1-r)}^{\theta} \frac{t-\varphi}{(1-r)^{2}+(t-\varphi)^{2}} d t \\
& =-\beta \int_{c(1-r)}^{\theta-\varphi} \frac{u}{(1-r)^{2}+u^{2}} d u=-\beta \int_{c}^{(\theta-\varphi) / 1-r} \frac{t}{1+t^{2}} d t \\
& =-\frac{\beta}{2} \log \left(\frac{1+((\theta-\varphi) /(1-r))^{2}}{1+c^{2}}\right) .
\end{aligned}
$$

From this, and a similar argument, we deduce

$$
\left|\frac{g\left(r e^{i \theta}\right)}{g\left(r e^{i(\varphi+c(1-r))}\right)}\right| \sim\left(\frac{1+c^{2}}{1+((\theta-\varphi) /(1-r))^{2}}\right)^{1 / 2 \beta},
$$

and

$$
\left|\frac{g\left(r e^{i \theta}\right)}{g\left(r e^{i(\varphi-c(1-r))}\right)}\right| \sim\left(\frac{1+c^{2}}{1+((\theta-\varphi) /(1-r))^{2}}\right)^{1 / 2 \beta}
$$

valid uniformly for $c(1-r)<\theta-\varphi<C(1-r)$, and $-C(1-r)<\theta-\varphi<$ $-c(1-r)$ respectively. When $|\theta-\varphi| \leqslant c(1-r)$ we have, using (2.15) again and Lemma 2.1(ii),

$$
|\log | \frac{g\left(r e^{i \theta}\right)}{g\left(r e^{i \varphi}\right)}||=\left|\int_{\varphi}^{\theta} \operatorname{Im} G\left(r e^{i t}\right) d t\right| \leqslant c(1-r)\left(1+\frac{K r}{1-r}\right),
$$

from which

$$
e^{-c(1-r+K r)} \leqslant\left|\frac{g\left(r e^{i \theta}\right)}{g\left(r e^{i \varphi}\right)}\right| \leqslant e^{c(1-r+K r),}
$$

and this modifies trivially to

$$
\begin{aligned}
e^{-c(1-r+K r)}\left(\frac{1}{1+((\theta-\varphi) /(1-r))^{2}}\right)^{1 / 2 \beta} & \leqslant\left|\frac{g\left(r e^{i \theta}\right)}{g\left(r e^{i \varphi}\right)}\right| \\
& \leqslant e^{c(1-r+K r)}\left(\frac{1+c^{2}}{1+((\theta-\varphi) /(1-r))^{2}}\right)^{1 / 2 \beta} .
\end{aligned}
$$

Since $c$ is an arbitrary positive number, the last two results imply (iii).

To conclude this sequence of lemmas we shall prove a result assumed in $\S 1$.

LEMMA 2.5. Let $g$ be a star-powered function of positive order $\beta$, and denote by $\psi_{1}, \ldots, \psi_{p}$ the values of $\psi$ in $[0,2 \pi)$ for which $\beta$ is the radial order of fon $\left\{r e^{i \varphi}\right\}$. Then 


$$
M(r, g) \sim \max \left(\left|g\left(r e^{i \psi_{1}}\right)\right|, \ldots,\left|g\left(r e^{i \psi_{p}}\right)\right| .\right.
$$

Proof. Let

$$
T(r)=[0,2 \pi] \backslash \bigcup_{k=1}^{p}\left\{\theta \bmod 2 \pi:\left|\theta-\psi_{k}\right|<l(r)\right\},
$$

then from Lemma 2.2, and the inequalities

$$
|g(z)| \geqslant r(1+r)^{\nu(2 \pi)-\nu(0)}\left|1-z e^{-i \psi} k\right|-\beta \quad(|z|=r, k=1, \ldots, p),
$$

which follows easily from (2.1), we have

$$
\sup _{\theta \in T(r)}\left|g\left(r e^{i \theta}\right)\right|=o(1) M(r, g) \text {. }
$$

Now using Lemma 2.3, we see via (2.16) that if $\epsilon>0$ and

then, for $r$ near one,

$$
W(r, \epsilon)=[0,2 \pi] \backslash \bigcup_{k=1}^{p}\left\{\theta \bmod 2 \pi:\left|\theta-\psi_{k}\right|<\epsilon(1-r)\right\},
$$

$$
\sup _{\theta \in W(r, \epsilon)}\left|g\left(r e^{i \theta}\right)\right|<\left(1+1 / 2 \epsilon^{2}\right)^{-1 / 2 \beta} M(r, g) .
$$

So $\left|g\left(r e^{i \eta(r)}\right)\right|=M(r, g)$ where, for the same $\epsilon$ and $r,\left|\eta(r)-\psi_{k}\right| \leqslant \epsilon(1-r)$ and $k=k(r) \in\{1, \ldots, N\}$. Since $\epsilon$ is an arbitrary positive number we deduce from Lemma 2.4(iii) that

where $k=k(r)$. Hence

$$
M(r, g)<(1+o(1))\left|g\left(r e^{i \psi k}\right)\right|
$$

$$
M(r, g)<(1+o(1)) \max \left(\left|g\left(r e^{i \psi_{1}}\right)\right|, \ldots,\left|g\left(r e^{i \psi} p\right)\right|\right),
$$

and obviously this completes the proof.

3. Proof of Theorem 1. Let $f$ be a starlike function of positive order $\alpha$, and denote by $\varphi_{1}, \ldots, \varphi_{N}$ the values of $\varphi$ in $[0,2 \pi)$ for which $\alpha$ is the radial order of $f$ on $\left\{r e^{i \varphi}\right\}$. Let $l(r)=(-\log (1-r))^{-1}$, and put

$$
\begin{gathered}
U_{k}(r)=\left\{\theta:\left|\theta-\varphi_{k}\right|<l(r)\right\} \quad(k=1 \text { to } N), \\
T(r)=[0,2 \pi] \backslash \bigcup_{k=1}^{N}\left\{\theta \bmod 2 \pi: \theta \in U_{k}(r)\right\} .
\end{gathered}
$$

Then, for real $\sigma, \tau$ and $k$, and $r$ near one, $H(r, \sigma, \tau, \kappa)=\Sigma_{k=1}^{N} X_{k}+Y$, where

$$
X_{k}=\int_{U_{k}(r)}|f|^{\sigma}|F|^{\tau}(\operatorname{Re} F)^{\kappa} d \theta, \quad Y=\int_{T(r)}|f|^{\sigma}|F|^{\tau}(\operatorname{Re} F)^{\kappa} d \theta .
$$

When $\alpha \sigma+\tau+\kappa>1, \tau+\kappa \geqslant 0, \kappa \geqslant 0, \sigma>0$, we shall find for each $X_{k}$ the asymptotic formula

$$
X_{k} \sim \alpha^{\tau+\kappa} C(\alpha \sigma+\tau+2 \kappa)(1-r)^{1-\tau-\kappa} \mid f\left(r e^{i \varphi k}\right)^{\mid \sigma}
$$


and for $Y$ the estimate

$$
Y=o(1)(1-r)^{1-\alpha \sigma-\tau-\kappa} .
$$

Since, by the representation (1.5), $\left|f\left(r e^{i \varphi_{k}}\right)\right| \geqslant r(1+r)^{-2}(1-r)^{-\alpha}$, we then have $Y=o(1) \Sigma_{k=1}^{N} X_{k}$; and consequently $H(r, \sigma, \tau, \kappa) \sim \sum_{k=1}^{N} X_{k}$. Our proof of (3.1) is in $\S 3.1$, and that of (3.2) in $\$ 3.2$.

3.1. Denote by $\varphi$ any one of the $\varphi_{k}$, and by $U(r)$ the corresponding $U_{k}(r)$. We have to prove

$$
\begin{aligned}
\int_{U(r)}\left|f\left(r e^{i \theta}\right)\right|^{\sigma}\left|F\left(r e^{i \theta}\right)\right|^{\tau}\left(\operatorname{Re} F\left(r e^{i \theta}\right)\right)^{\kappa} d \theta & \\
& \sim \alpha^{\tau+\kappa} C(\alpha \sigma+\tau+2 \kappa)(1-r)^{1-\tau-\kappa} \mid f\left(r e^{i \varphi}\right)^{\mid \sigma}
\end{aligned}
$$

for $\alpha \sigma+\tau+\kappa>1, \tau+\kappa \geqslant 0, \kappa \geqslant 0, \sigma>0$. Let

$$
V(r, x)=\{\theta:|\theta-\varphi|<x(1-r)\} \quad(x>0)
$$

and write

$$
\int_{U(r)}=\int_{V(r, x)}+\int_{U(r) \backslash V(r, x)}=I_{1}+I_{2} \text {, }
$$

say, where the missing integrand is that in (3.3). For $I_{1}$ we have, by Lemma 2.4, for real $\sigma, \tau$, and $\kappa$, and for $x>0$,

$$
\begin{array}{r}
I_{1} \sim \alpha^{\tau+\kappa}(1-r)^{-\tau-\kappa}\left|f\left(r e^{i \varphi}\right)\right|^{\sigma} \int_{V(r, x)}\left(1+\left(\frac{\theta-\varphi}{1-r}\right)^{2}\right)^{-1 / 2(\alpha \sigma+\tau+2 \kappa)} d \theta \\
=\alpha^{\tau+\kappa}(1-r)^{1-\tau-\kappa}\left|f\left(r e^{i \varphi}\right)\right|^{\sigma} \int_{-x}^{x}\left(1+t^{2}\right)^{-1 / 2(\alpha \sigma+\tau+2 \kappa)} d t .
\end{array}
$$

For $I_{2}$ consider first the case $\tau+\kappa \neq 0$. Let $p$ and $q$ be chosen so that $\alpha o p>1$, $(\tau+\kappa) q>1, p^{-1}+q^{-1}=1, p>1$; it is easy to verify that this is possible.

Then, using the inequality $k \geqslant 0$, and Hölder's inequality, we obtain

$$
I_{2} \leqslant \int_{U V V}|f|^{\sigma}|F|^{\tau+\kappa} d \theta \leqslant\left(\int_{U V V}|f|^{\sigma p}\right)^{1 / p}\left(\int_{0}^{2 \pi}|F|^{(\tau+\kappa) q}\right)^{1 / q},
$$

where $U \equiv U(r), V \equiv V(r, x)$. With $I(\lambda)=\int_{U \backslash V}|f|^{\lambda}$, and use of Hayman's wellknown estimate [1], applicable here since $(\tau+\kappa) q>1$, this becomes

$$
I_{2}=O(1)(1-r)^{(1 / q)-\tau-\kappa}(I(\sigma p))^{1 / p} .
$$

To deal with $I$ we put

$$
g(z)=z\left(1-z e^{-i \varphi}\right)^{1 / 2(\alpha \lambda+1)}(f(z) / z)^{\lambda} \quad(z \in D \backslash\{0\})
$$

where $\alpha \lambda>1$, and write

$$
\begin{aligned}
r^{1-\lambda} I(\lambda) & =\int_{U V V^{\prime}} \lg \left(r e^{i \theta}\right) \| 1-r e^{\left.i(\theta-\varphi)\right|^{-1 / 2(\alpha \lambda+1)} d \theta} \\
& =\int_{\varphi-l(r)}^{\varphi-x(1-r)}+\int_{\varphi+x(1-r)}^{\varphi+l(r)}=J_{1}+J_{2} .
\end{aligned}
$$


On $\left\{r e^{i \varphi}\right\} g$ has radial order $1 / 2(\alpha \lambda-1)>0$, so the lemmas of $\$ 2$ apply. From Lemma 2.4(ii) and (2.15), we see that, for $r$ near one, $\left|g\left(r e^{i \theta}\right)\right|$ increases throughout the interval $\left(\varphi-x^{\prime}(1-r), \varphi-x(1-r)\right)$. So on applying Lemma 2.3 to $\left|g\left(r e^{i \theta}\right)\right|$ for $\theta$ in $\left(\varphi-l(r), \varphi-x^{\prime}(1-r)\right)$ we have for $r>r_{0}(x)$ that in $(\varphi-l(r)$, $\varphi-x(1-r)),\left|g\left(r e^{i \theta}\right)\right|<\left|g\left(r e^{i(\varphi-x(1-r))}\right)\right|$. This result applied to $J_{1}$, and a similar one applied to $J_{2}$, give for $r>r_{0}(x)$

$$
r^{1-\lambda} I(\lambda) \leqslant \max \left|g\left(r e^{i(\varphi \pm x(1-r))}\right)\right| \int_{U \backslash V}\left|1-r e^{i(\theta-\varphi)}\right|^{-1 / 2(\alpha \lambda+1)} d \theta .
$$

Now using Lemma 2.4(iii) we obtain, for $r>r_{0}(x)$,

$$
I(\lambda)<\left|g\left(r e^{i \varphi}\right)\right| \int_{U \backslash V} \mid 1-r e^{i(\theta-\varphi) \mid-1 / 2(\alpha \lambda+1)} d \theta .
$$

For $\epsilon>0, x$ suitably large, and $r$ near one, it is easy to prove that

$$
\int_{U \backslash V}\left|1-r e^{i(\theta-\varphi) \mid-1 / 2(\alpha \lambda+1)} d \theta<\epsilon \int_{V}\right| 1-r e^{i(\theta-\varphi) \mid-1 / 2(\alpha \lambda+1)} d \theta
$$

by making the substitution $\theta-\varphi=t(1-r)$ and using $\alpha \lambda>1$. Moreover, for any given positive $x$, and $r$ near one,

$$
\begin{aligned}
\int_{V} \mid 1-r e^{i(\theta-\varphi) \mid-1 / 2(\alpha \lambda+1)} d \theta & \leqslant \int_{0}^{2 \pi} \mid 1-r e^{i(\theta-\varphi) \mid-1 / 2(\alpha \lambda+1)} d \theta \\
& <A(1-r)^{-1 / 2(\alpha \lambda-1)}
\end{aligned}
$$

where $A$ denotes an absolute constant. So when $x>x_{0}(\epsilon)$ and $r>r_{0}(\epsilon)$ we have

$$
I(\lambda)<\left.A \epsilon \lg \left(r e^{i \varphi}\right)\left|(1-r)^{-1 / 2(\alpha \lambda-1)}<A \epsilon(1-r)\right| f\left(r e^{i \varphi}\right)\right|^{\lambda}
$$

for $\alpha \lambda>1$, and since $\alpha \sigma p>1$ we may substitute (3.7) into (3.6) with $\lambda=\sigma p$ and obtain, for any $\epsilon>0$,

(3.8) $I_{2}<A \epsilon(1-r)^{1-\tau-\kappa}\left|f\left(r e^{i \varphi}\right)\right|^{\sigma} . \quad\left(x>x_{0}(\epsilon), r>r_{0}(\epsilon)\right)$.

To prove (3.8) when $\tau+\kappa=0$ we first deduce $I_{2} \leqslant I(\sigma)$ using $\kappa \geqslant 0$, and then note that (3.7) applies with $\lambda=\sigma$ since $\alpha \sigma=\alpha \sigma+\tau+\kappa>1$. Now using (3.4), (3.5) and (3.8) we easily obtain (3.3).

3.2. We have to prove that for $\alpha \sigma+\tau+\kappa>1, \tau+\kappa \geqslant 0, \kappa \geqslant 0, \sigma>0$,

$$
\int_{T(r)}\left|f\left(r e^{i \theta}\right)\right|^{\sigma}\left|F\left(r e^{i \theta}\right)\right|^{\tau}\left(\operatorname{Re} F\left(r e^{i \theta}\right)\right)^{\kappa} d \theta=o(1)(1-r)^{1-\alpha \sigma-\tau-\kappa} .
$$

Assume $\tau+\kappa>0$ so that $p$ and $q$ exist satisfying $\alpha \sigma p>1,(\tau+\kappa) q>1$, $p^{-1}+q^{-1}=1, p>1$. Then, since $k \geqslant 0$, we have by Hölder's inequality

$$
\begin{aligned}
\int_{T(r)}|f|^{\sigma}|F|^{\tau}(\operatorname{Re} F)^{\kappa} & \leqslant\left(\int_{T(r)}|f|^{\sigma p}\right)^{1 / p}\left(\int_{0}^{2 \pi}|F|^{(\tau+\kappa) q}\right)^{1 / q} \\
& =O(1)(1-r)^{1 / q-\tau-\kappa}\left(\int_{T(r)}|f|^{\sigma p}\right)^{1 / p},
\end{aligned}
$$


where we have used Hayman's result [1], applicable since $(\tau+\kappa) q>1$.

We now let $\lambda=\sigma \rho$, so that $\alpha \lambda>1$, and consider $\int_{T(r)}|f|^{\lambda} d \theta$. Denote by $\varphi_{1}, \ldots, \varphi_{N}, \ldots$ the sequence of $\varphi$ for which on $\left\{r e^{i \varphi}\right\}$ the radial order of $f$ is positive, and by $\alpha_{k}$ the radial order of $f$ on $\left\{r e^{i \varphi_{k}}\right\}$. Then, since $\alpha \lambda>1$, we define a star-powered function $g$ by

$$
g(z)=z\left(\frac{f(z)}{z}\right)^{\lambda} \prod_{0<\gamma_{k}<1}\left(1-z e^{-i \varphi_{k}}\right)^{\gamma_{k}+\xi} \quad(z \in D \backslash\{0\})
$$

where $\gamma_{k}=\alpha_{k} \lambda-\alpha \lambda+1, \xi=\min \left(\alpha \lambda-1,1-\gamma_{k}\right)$.

Any radial order of $g$ less than the order is also less than $\alpha \lambda-1$. Thus, by Lemma 2.2, for some positive $\delta$,

$$
\sup _{\theta \in T(r)}\left|g\left(r e^{i \theta}\right)\right|=O(1)(1-r)^{1-\alpha \lambda+\delta} .
$$

Hence

$$
\begin{aligned}
\int_{T(r)}|f|^{\lambda} d \theta & =O(1)(1-r)^{1-\alpha \lambda+\delta} \int_{0}^{2 \pi} \prod_{0<\gamma_{k}<1}\left|1-r e^{i\left(\theta-\varphi_{k}\right)}\right|^{-\gamma_{k}-\xi} d \theta \\
& =O(1)(1-r)^{1-\alpha \lambda+\delta} \int_{0}^{2 \pi}\left|1-r e^{i \theta}\right|^{-1} d \theta,
\end{aligned}
$$

since $\gamma_{k}+\xi \leqslant 1$, and the points $\varphi_{k}$ for which $0 \leqslant \gamma_{k}<1$ are distinct and finite in number. The last integral is $O(\log 1 /(1-r))$, so

$$
\int_{T(r)}|f|^{\lambda} d \theta=o(1)(1-r)^{1-\alpha \lambda}
$$

and (3.9) follows easily from this (with $\lambda=\sigma p$ ) and (3.10). To prove (3.9) when $\tau+\kappa=0$, we first use $\kappa \geqslant 0$ to deduce

$$
\int_{T(r)}|f|^{\sigma}|F|^{\tau}(\operatorname{Re} F)^{\kappa} d \theta \leqslant \int_{T(r)}|f|^{\sigma} d \theta
$$

and then note that (3.11) applies with $\lambda=\sigma$, since $\alpha \sigma=\alpha \sigma+\tau+\kappa>1$.

4. Proof of a conjecture by Holland and Thomas. With the aid of a corollary to Theorem 1, we can outline a proof of the conjecture (1.10) in the case $\alpha>0$, that is (in the notation of (1.10))

$$
\lim _{r \rightarrow 1} \frac{(1-r) J^{\prime}(r, \lambda)}{J(r, \lambda)}=(1+\alpha) \lambda-1, \text { for } \alpha>0,(1+\alpha) \lambda>1
$$

where

$$
J(r, \lambda)=\frac{1}{2 \pi r^{\lambda}} \int_{0}^{2 \pi} \mid f\left(r e^{i \theta}\right) F\left(r e^{i \theta}\right)^{\lambda} d \theta .
$$

In proving a similar result for $I$ (also conjectured by Holland and Thomas), we used Theorem 1 to represent both $I$ and $I^{\prime}$. The same approach will suffice for $J$ but not for $J^{\prime}$ since we have 


$$
J^{\prime}(r, \lambda)=\frac{\lambda}{2 \pi r^{\lambda+1}} \int_{0}^{2 \pi}\left|f\left(r e^{i \theta}\right) F\left(r e^{i \theta}\right)\right|^{\lambda}\left(\operatorname{Re} F\left(r e^{i \theta}\right)+\operatorname{Re} F\left(r e^{i \theta}\right)-1\right) d \theta
$$

where

$$
F(z)=z F^{\prime}(z) / F(z) \quad(z \in D) .
$$

However, when $(1+\alpha) \lambda>1$, a representation of $J^{\prime}$ can be squeezed out from the proof of Theorem 1.

The following results on $F$ are needed:

(i) If $f$ has positive radial order on $\left\{r e^{i \varphi}\right\}$

$$
\operatorname{Re} F\left(r e^{i \theta}\right) \sim\left(1+\left(\frac{\theta-\varphi}{1-r}\right)^{2}\right)^{-1 / 2}(1-r)^{-1}
$$

uniformly when $|\theta-\varphi|<O(1)(1-r)$.

$$
|F(z)| \leqslant 2 r /\left(1-r^{2}\right), \quad(|z|=r) .
$$

(i) follows from an argument similar to that in Lemma 2.4(i); for (ii) see [3]. Next we have the

COROLlaRY to THEOREM 1. Let $f$ and $\varphi_{1}, \ldots, \varphi_{N}$ be given as in Theorem 1 , and suppose that $\sigma>0, \tau \geqslant 0, \alpha \sigma+\tau>1$. If $\Phi$ is any real function on $D$ for which

$$
\Phi\left(r e^{i \theta}\right) \sim\left(1+\left(\frac{\theta-\varphi_{k}}{1-r}\right)^{2}\right)^{-1 / 2}(1-r)^{-1}
$$

uniformly when $\left|\theta-\varphi_{k}\right|<O(1)(1-r)$, and

$$
|\Phi(z)|=O(1)(1-r)^{-1} \quad(|z|=r),
$$

then

$$
\int_{0}^{2 \pi}\left|f\left(r e^{i \theta}\right)\right|^{\sigma}\left|F\left(r e^{i \theta}\right)\right|^{\tau} \Phi\left(r e^{i \theta}\right) d \theta \sim \alpha^{\tau} C(\alpha \sigma+\tau+1)(1-r)^{-\tau} \sum_{k=1}^{N} \mid f\left(r e^{i \varphi k}\right)^{\sigma} .
$$

Clearly we may take $\Phi=\operatorname{Re} F$ in the corollary. In this way we find a representation for

$$
\int_{0}^{2 \pi}\left|f\left(r e^{i \theta}\right) F\left(r e^{i \theta}\right)\right|^{\lambda} \operatorname{Re} F\left(r e^{i \theta}\right) d \theta
$$

when $\alpha>0,(1+\alpha) \lambda>1$. Theorem 1 supplies representations for

$$
\int_{0}^{2 \pi}\left|f\left(r e^{i \theta}\right) F\left(r e^{i \theta}\right)\right|^{\lambda} \operatorname{Re} F\left(r e^{i \theta}\right) d \theta
$$

when $\alpha>0, \lambda>0$, and for $J(r, \lambda)$ when $\alpha>0,(1+\alpha) \lambda>1$. With these results it is not difficult to obtain (4.1) via (4.2) and (4.3).

\section{REFERENCES}

1. W. K. Hayman, On functions with positive real part, J. London Math. Soc. 36 (1961), 35-48. MR 27 \#311. 
2. F. Holland and D. K. Thomas, On the order of a starlike function, Trans. Amer. Math. Soc. 158 (1971), 189-201. MR 43 \#3438.

3. R. R. London and D. K. Thomas, An area theorem for starlike functions, Proc. London Math. Soc. (3) 20 (1970), 734-748. MR 41 \#7087.

4. Ch. Pommerenke, On starlike and convex functions, J. London Math. Soc. 37 (1962), 209-224. MR 25 \#1279.

5. T. Sheil-Small, Starlike univalent functions, Proc. London, Math. Soc. (3) 21 (1970), 577-613. MR 43 \#2207.

DEPARTMENT OF PURE MATHEMATICS, UNIVERSITY COLLEGE OF SWANSEA, SWANSEA SA2 8PP, WALES 